\title{
Acoustic Channel Modeling and Simulation for Underwater Acoustic Wireless Sensing Networks
}

\author{
Garima Gupta \\ M.Tech Research Scholar CSE \\ H.C.T.M College, Kaithal \\ Kaithal (Haryana), India
}

\author{
Rajender Kumar \\ Assistant Professor CSE \\ H.C.T.M College, Kaithal \\ Kaithal (Haryana), India
}

\begin{abstract}
Underwater acoustic channels are band-limited and reverberant, posing many obstacles to reliable, phase-coherent acoustic communications. In terrestrial wireless sensor networks, the nodes use radio frequency (RF) to build up the communication. In underwater environments, due to water absorption, radio wave does not work well. Compared to radio waves, sound has superior propagation characteristics in water, making it the preferred technology for underwater communications. While many high frequency communication experiments have been conducted in shallow water, few have carried out systematic studies on the channel properties at a time scale relevant for communications.

This Research work describes the unique characteristics of the underwater environment and its effects on the design of UWSN. In addition, the differences between terrestrial WSN and UWSN are presented. Even though they are different, terrestrial WSN is still valuable on UWSN. Major challenges including power consumption, communication techniques, and routing protocols are discussed. These aspects are vital important to do future research on UWSN, especially developing new generation UWSN.
\end{abstract}

\section{Keywords}

RF, UWSN, WSN, UAWSN.

\section{INTRODUCTION}

The earth is a water planet. The largely unexplored vastness of the ocean, covering about two-thirds of the surface of Earth, has fascinated humans for as long as we have records. Recently, there has been a growing interest in monitoring aqueous environments (including oceans, rivers, lakes, ponds and reservoirs, etc.) for scientific exploration, commercial exploitation and attack protection. The ideal vehicle for this type of extensive monitoring is a networked underwater wireless. sensor distributed system, referred to as Underwater Acoustic Wireless Sensor Network (UAWSN).

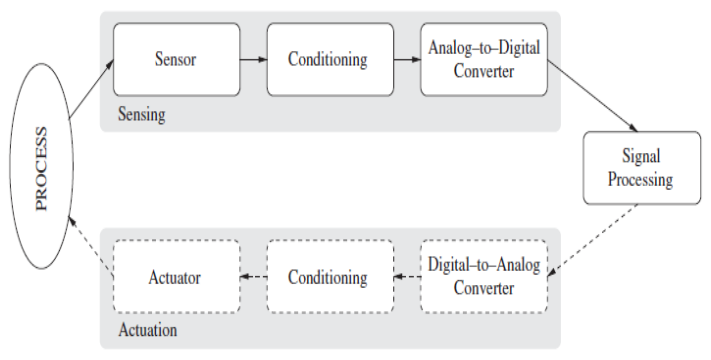

Figure 2: General Architecture of a wireless sensor [1] actuators that permit them to undeviatingly manipulation the

physical world. For example, an actuator can be a valve manipulating the flow of hot water, a motor that opens or closes a door or window, or a impel that controls the number of gas inoculated into an engine. Such a wireless sensor and actuator web (WSAN) seizes commands from the processing mechanism (controller) and transforms these commands into input signals for the actuator, that next interacts alongside a physical procedure, thereby growing a closed manipulation loop.

\section{LITERATURE REVIEW}

Chitre and Pelekanakisy[4] conclude that stronger arrivals usually vary less (in relative terms) as compared to weaker arrivals. At longer timescales, they find that the direct arrival is typically the less variable (more stable) although it may not always be the strongest.The average short timescale variability of all arrivals is about the same, but varies significantly across links.

\section{CHALLENGES POSED BY THE CHANNEL CHARACTERISTICS OF UNDERWATER ACOUSTIC CHANNELS}

A few characteristics of the underwater acoustic channel is described in the next few subsections.

\subsection{Doppler shift}

A relative motion of the receiver and or the transmitter or a moving medium can change the frequency of the sound waves propagating through the channel. The apparent change in the signal's carrier frequency and the time domain is known as Doppler shift. An expression for the Doppler frequency shift is given by:

$\mathrm{f}_{\mathrm{d}}=\mathrm{f}_{\mathrm{c}} \frac{v+c}{c}$

where $\mathrm{c}$ is the speed of sound in free space, $\mathrm{v}$ speed of the observer and $\mathrm{f} \mathrm{c}$ the transmitted signal frequency known as the carrier frequency.

\subsection{Multipath}

In underwater acoustics the multipath effect is mainly caused by reflections from the seafloor and surface. The number of bounces determines the multipath spread. In addition, the channel consists of volume reflections such as plankton and fish. For a large enough range between the transmitter and receiver, the transmitted signal propagates to the receiver via various paths. The delay associated with each path depends on its geometry. 


\subsection{Delay-Spread and Doppler-Spread Functions}

The convolution theorem gives the input-output relationship that exists between the input signal $\mathrm{x}(\mathrm{t})$ and output signal $\mathrm{y}(\mathrm{t})$ as:

$$
\mathrm{Y}(\mathrm{t})=\int \boldsymbol{x}(\boldsymbol{t}-\boldsymbol{\tau}) \boldsymbol{h}(\boldsymbol{t}, \boldsymbol{\tau}) d \boldsymbol{\tau},
$$

This shows that the output of the channel is expressed as the integral of the delay elements; with the elements providing delays in the interval $(\boldsymbol{\tau}, \boldsymbol{\tau}+\mathrm{d} \boldsymbol{\tau})$ and having a differential amplitude of $\mathrm{h}(\mathrm{t}, \boldsymbol{\tau}) \mathrm{d} \boldsymbol{\tau}$. Therefore, the function $\mathrm{h}(\mathrm{t}, \boldsymbol{\tau})$ is called the Input Delay-Spread Function of the channel. The second expression for the output signal is obtain by applying the delay operator at the output of the channel; the required expression is given by:

$$
\mathrm{Y}(\mathrm{t})=\int \boldsymbol{x}(\boldsymbol{t}-\boldsymbol{\tau}) \boldsymbol{g}(\boldsymbol{t}-\boldsymbol{\tau}, \boldsymbol{\tau}) \mathrm{d \tau}
$$

where $\mathrm{g}(\mathrm{t}, \boldsymbol{\tau})=\mathrm{h}(\mathrm{t}+\boldsymbol{\tau}, \boldsymbol{\tau})$ is referred to as the Output DelaySpread-Function of the channel and may be thought of as the response seconds in the future to a unit impulse at time t. For the same requirement imposed on $\mathrm{h}(\mathrm{t}, \boldsymbol{\tau}), \mathrm{g}(\mathrm{t}, \boldsymbol{\tau})$ must vanish for $\boldsymbol{\tau}<0$.

\subsection{Delay-Doppler-Spread and Doppler- Delay-Spread Functions$$
\mathrm{h}(\mathrm{t}, \boldsymbol{\tau})=\int U(\tau-\rho) e^{j 2 \pi p t} d \rho,
$$

Substituting this expression into $\mathrm{y}(\mathrm{t})$ gives:

$$
\mathrm{y}(\mathrm{t})=\iint \boldsymbol{x}(\boldsymbol{t}-\boldsymbol{\tau}) \boldsymbol{U}(\boldsymbol{\tau}-\boldsymbol{\rho}) \boldsymbol{e}^{j 2 \pi p t} \boldsymbol{d} \boldsymbol{p d \tau},
$$

this shows that the output of the channel is expressed as the sum of the delay and then Doppler shifted elements; with the elements providing delays in the interval $(\boldsymbol{\tau}, \boldsymbol{\tau}+\mathrm{d})$ and Doppler shifts in the interval $(\boldsymbol{\rho}, \boldsymbol{\rho}+\mathrm{d} \boldsymbol{\tau})$ and having a differential amplitude of $\mathrm{U}(\boldsymbol{\tau}, \boldsymbol{\rho}) \mathrm{d} \boldsymbol{\rho} \mathrm{d} \boldsymbol{\tau}$. Hence, the function $\mathrm{U}(\boldsymbol{\tau}, \boldsymbol{\rho})$ is called the Delay-Doppler-Spread Function of the channel.

To determine the Doppler-Delay-Spread function of the channel, we first express the input Doppler-Spread Function as the Fourier transform of $\mathrm{V}(\boldsymbol{\rho}, \boldsymbol{\tau})$ (holding $\boldsymbol{\rho}$ as a fixed parameter)

$$
\mathrm{H}(\mathrm{f}, \rho)=\int v(\rho, \tau) e^{-j 2 \pi \tau t} d \tau,
$$

Substituting this expression into Equation 2.23 gives:

$$
\mathrm{y}(\mathrm{f})=\iint x(f-\rho) V(\rho, \tau) e^{-j 2 \pi \tau f} d r d \rho
$$

The output of the channel is expressed as the sum of the Doppler shifted and then Delay elements; with the elements providing Doppler shifts in the interval $\boldsymbol{\rho}, \boldsymbol{\rho}+\mathrm{d} \boldsymbol{\rho})$ and delays in the interval $(\boldsymbol{\tau}, \boldsymbol{\tau}+\mathrm{d} \boldsymbol{\tau})$ and having a differential amplitude of $\mathrm{V}(\boldsymbol{\rho}, \boldsymbol{\tau}) \mathrm{d} \boldsymbol{\tau} \mathrm{d} \boldsymbol{\rho}$. For this reason, the function $\mathrm{V}(\boldsymbol{\rho}, \boldsymbol{\tau})$ is called the Doppler-Delay-Spread Function of the channel.

\section{RESULT}

The basic (time-invariant, deterministic) model of an acoustic channel is that of a multipath channel with additional lowpass filtering. Low-pass filtering accounts for energy absorption which is higher for higher acoustic frequencies. The signal also attenuates with distance, according to the energy spreading law (quadratic with distance for spherical geometry of spreading, linear for cylindrical).

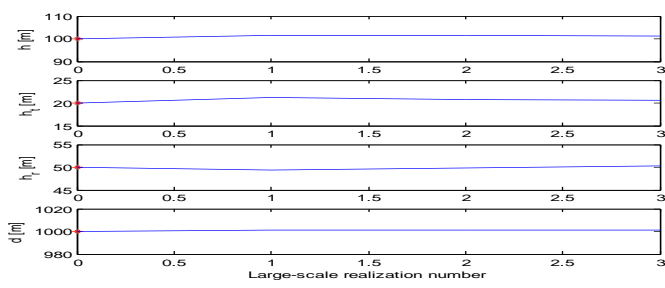

Figure 2. UnLarge Scale Realization number with respect to height [meters]

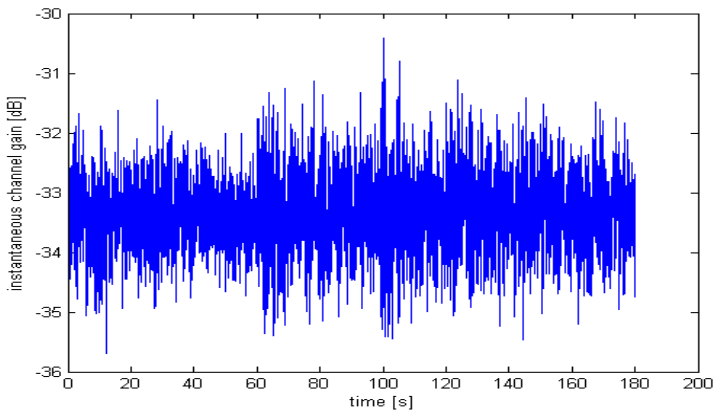

Figure 3. Instantaneous Channel gain is described with respect to time where channel distance $\mathrm{d} 0=\mathbf{1 0 0 m}$

Due to the absorption, properties of sound waves in water, energy spreading law and Doppler Effect we see delays and gains in the simulated channel one such instantaneous Channel gain is described with respect to time where channel distance $\mathrm{d} 0=100 \mathrm{~m}$.

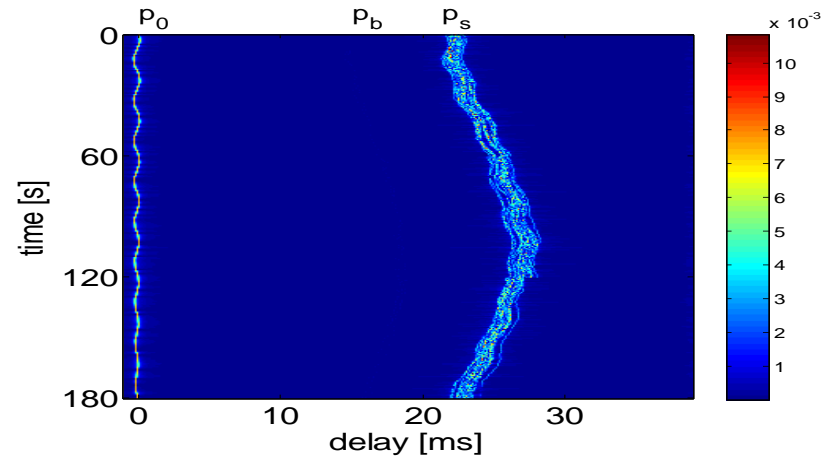

Figure 4. hows the effect of raising the distance $d_{0}=100$ to $d_{0}=150$, just by 50 meters the delay is greatly reduced but still it is not good for communication.

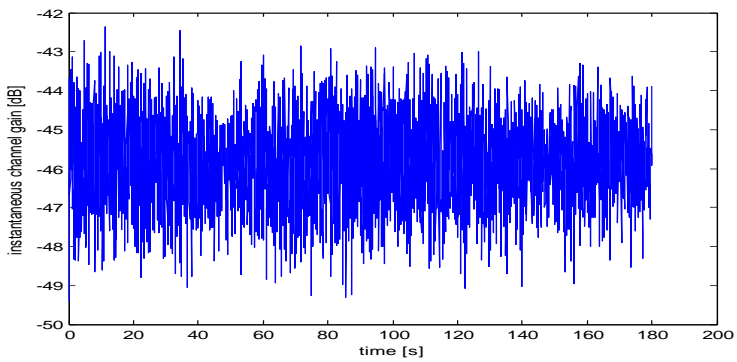

Figure 5. Shows the channel gain at the channel range at $d_{0}=300 \mathrm{~m}$, the channel gain is improved from $-35 \mathrm{db}$ to 46db. 


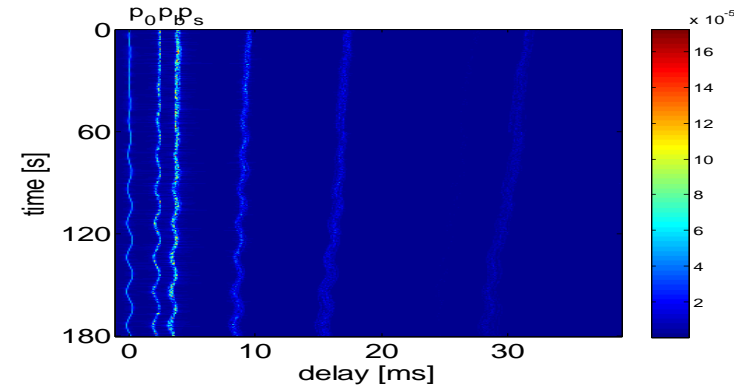

Figure 6. Shows the effect of raising the distance $d_{0}=1000 \mathrm{~m}$, by increasing the channel distance delay is improved throughout of the propagation time.

So it can be concluded that underwater channel communication at this range is better than $250 \mathrm{~m}$ range but we can see lot of reflections of the propagated signal.

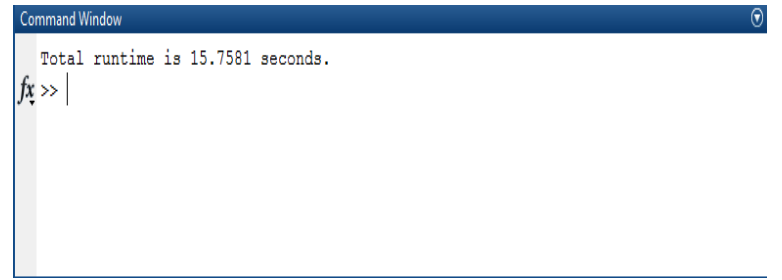

Figure 7. Total Simulation time

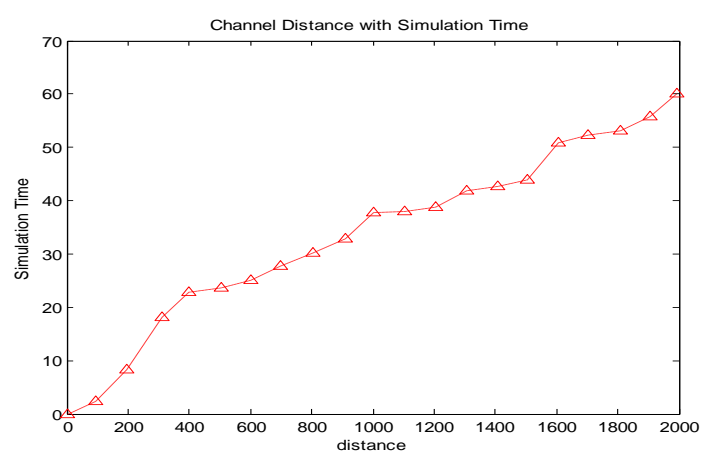

Figure 8. Shows the simulation time required for running the underwater simulator, the $x$ axis shows the channel distance do from ranges 200-2000 meters.
It can be concluded that the best channel distance ranges from $250 \mathrm{~m}-300 \mathrm{~m}$ for underwater acoustic communications, especially at $300 \mathrm{~m}+$ range, at this range we see low channel delay and spreading between the underwater channels. so it is recommended by our research to select this channel distance for better communication.

\section{CONSLUSION AND FUTURE WORK}

The Research is being conducted by the various researchers world-wide concerning on key question, that is, how to effectively model underwater channel? As we must take into account various physical aspects of acoustic propagation as well as the effects of inevitable random channel variations. The underwater signal also attenuates with distance, according to the energy spreading law. Hence to deploy underwater nodes the basic (time-invariant, deterministic) model of an acoustic channel is that of a multipath channel is required. As the actual deployment of UAWSN Nodes without proper channel model is impossible, in future the work will try to simulate UAWSN Channel taking into consideration highly dynamic and almost chaotic nature of ocean.

\section{REFERENCES}

[1] Waltenegus, D., and Poellabauer, C.,(2011)," Fundamentals of wireless sensor networks: theory and practice", John Wiley \& Sons.

[2] Hill, J.L.,(2003),"System architecture for wireless sensor networks".

[3] Lewis, F.L., (2004)"Wireless sensor networks: Smart environments: technologies, protocols, and applications".

[4] Chitre, M., and Pelekanakisy, K.,(2013)" Channel variability measurements in an underwater acoustic network". 